\title{
The effect of kynurenic acid on the synthesis of selected cytokines by murine splenocytes - in vitro and ex vivo studies
}

\author{
JOANNA MAŁACZEWSKA ${ }^{1}$, ANDRZEJ K. SIWICKII, ROMAN M. WÓJCIK', \\ WALDEMAR A. TURSKI ${ }^{2}$ EDYTA KACZOREK ${ }^{1}$
}

'Department of Microbiology and Clinical Immunology, Faculty of Veterinary Medicine, University of Warmia and Mazury in Olsztyn, Poland ${ }^{2}$ Department of Experimental and Clinical Pharmacology, Medical University of Lublin, Poland

\begin{abstract}
Kynurenic acid (KYNA), a secondary product of the kynurenine pathway of tryptophan degradation, known mainly as an endogenous neuroprotectant, shows also immunotropic properties. Some quantities of KYNA are present in food and are effectively absorbed in the gastrointestinal tract. Since the spleen is an important target of dietary immunomodulators, the aim of the study was to determine the effect of exogenous KYNA on murine splenocytes. Splenocytes isolated from adult BALB/c mice were used in the study. Firstly, the effect of increasing KYNA concentrations $(0-5 \mathrm{mM})$ on the viability, and proliferative and cytokine response (interleukin $1 \beta[I L-1 \beta], I L-6, I L-10$, tumor necrosis factor $\alpha[T N F-\alpha])$ of murine splenocytes under in vitro conditions was determined. Then, proliferative and cytokine responses were determined in cells derived from animals receiving kynurenic acid in drinking water at concentrations of 2.5 , 25, or $250 \mathrm{mg} / \mathrm{l}$ for 7-14 days. Cytokine levels were measured using commercial immunoassay (ELISA) kits, and cell viability and proliferation was determined with MTT reduction assay. Exogenous KYNA was characterised by a low level of cytotoxicity towards murine splenocytes, and was well tolerated by the animals receiving it in drinking water. As expected, it exhibited anti-inflammatory action towards the activated splenocytes, under both in vitro and ex vivo conditions. Surprisingly, however, KYNA itself influenced the activity of resting, non-stimulated cells, exerting an immunostimulant effect in vitro, and an immunosuppressive effect under ex vivo conditions. The obtained results indicate not only anti-inflammatory, but also more complex, immunomodulating properties of KYNA, which require more detailed investigation.
\end{abstract}

Key words: kynurenic acid, cytokine response, murine splenocytes, immunomodulating properties.

(Cent Eur J Immunol 2016; 41 (1): 39-46)

\section{Introduction}

Kynurenic acid (KYNA) is a secondary product of the kynurenine pathway of tryptophan degradation, known mainly as an antagonist of ionotropic glutamate receptors and alpha-7 nicotinic receptor, and an endogenous neuroprotectant [1]. However, it has been known for some time that KYNA also has immunotropic properties. Under physiological conditions, its concentration in the peripheral blood and tissues does not exceed nanomolar values; however, it increases considerably in the course of infections and inflammatory processes due to increased degradation of tryptophan along the kynurenine pathway, stimulated by inflammatory mediators such as bacterial LPS, free radicals, or proinflammatory cytokines. Some of the metabolites produced on the kynurenine pathway, including
KYNA, subsequently exert an anti-inflammatory effect through negative feedback [1-6]. Kynurenic acid is the most important agonist of the orphan G-protein-coupled receptor (GPR35) identified so far, which comes to expression on various types of cells associated with the immune system, i.e. monocytes, T and B lymphocytes, neutrophils, dendritic cells, eosinophils, basophils, and NKT cells. The effect of stimulation of this receptor in inflammatory processes by the higher than physiological, micromolar concentrations of KYNA is a reduction in the synthesis of proinflammatory cytokines, nitric oxide, and reactive oxygen species (ROS) in the immune cells $[3,4$, 7-11]. More recent studies indicate that another target of the effect of KYNA on the immune system cells may be the aryl hydrocarbon receptor (AHR) present on their sur-

Correspondence: Joanna Małaczewska, Department of Microbiology and Clinical Immunology, Faculty of Veterinary Medicine, University of Warmia and Mazury in Olsztyn, Oczapowskiego 13, 10-718 Olsztyn, Poland, e-mail: j.malaczewska7@wp.pl Submitted: 11.12.2015; Accepted: 4.01.2016 
faces, which is involved in the metabolism of xenobiotics and regulation of the immune response. KYNA turns out to be one of the non-toxic agonists of this receptor, whereas for the activation of the AHR, at least for certain cell types, the physiological, nanomolar concentrations of the acid are sufficient. However, reports concerning the effect of the action of KYNA on this receptor are not consistent $[12,13]$.

In addition to KYNA being formed naturally in the body along the kynurenine pathway, some quantities thereof may come from external sources and enter the body with consumed foodstuffs. Relatively high KYNA concentrations are found in, inter alia, honeybee products and certain medicinal plants, including those traditionally used in the treatment of rheumatoid arthritis. Exogenous KYNA is effectively absorbed in the gastrointestinal tract; however, it is not metabolised but excreted, primarily with the urine [14-18]. Therefore, KYNA attracts justified interest as a natural, easy to apply immunomodulator correcting the inflammatory response of the body, and maybe even a potential dietary supplement with immunotropic properties.

Since one of the targets of a substance with immunotropic properties getting into the body through the alimentary route is the spleen, the aim of this study was to determine the effect of exogenous KYNA on the synthesis of cytokines by unstimulated murine splenocytes, and the proliferative and cytokine response of mitogen-stimulated splenocytes. Firstly, the effect of KYNA on the tested parameters under in vitro conditions was determined by incubating the isolated cells with increasing concentrations $(0,5,50,125,250 \mu \mathrm{M} ; 0.5,1.25,2.5$, and $5 \mathrm{mM})$. Then, the same parameters were determined in splenocytes derived from animals receiving KYNA in drinking water at concentrations of $2.5,25$, or $250 \mathrm{mg} / \mathrm{l}$ for a period of 7 or 14 days.

\section{Material and methods}

\section{Mice}

The study was performed on 96 male BALB/c mice, aged 10-12 weeks, with body weight of 22-26 g. Animals were kept on a 12-hour light/dark cycle in a controlled temperature $\left(20 \pm 1^{\circ} \mathrm{C}\right)$ with free access to food and water throughout the experiment. All experimental procedures were carried out in accordance with the guidelines of the European Communities Council Directive of November 1986 (86/609/EEC) and approved by the Local Ethics Committee for Animal Experimentation in Olsztyn, Poland.

For the in vitro experiment 48 animals were randomly divided into eight equal groups of six, anaesthetised by inhalation of AErrane (isofluranum, Baxter Poland), and their spleens were sampled after bleeding. Splenocytes iso- lated from the individuals within each group were pooled before performing the assays and cultured in media supplemented with KYNA (Sigma-Aldrich) within a range of concentrations from 0 (control) to $5 \mathrm{mM}$ for 48 or 72 hours.

The in vivo experiment was performed on 48 mice, randomly divided into four equal groups: a control group (0) not receiving the KYNA, and three experimental groups administered the KYNA solution in drinking water at the concentrations of $2.5,25$, or $250 \mathrm{mg} / \mathrm{l}$. After 7 and 14 consecutive days of administration of the KYNA solution, six animals from each group were sacrificed and their spleens were sampled after bleeding.

\section{Isolation of splenocytes}

The spleens were removed aseptically and pressed through a 60- $\mu$ m nylon mesh in RPMI-1640 medium with L-glutamine and sodium bicarbonate (Sigma-Aldrich). The splenocyte cell suspensions were placed on density gradient Histopaque 1077 (Sigma-Aldrich) in order to isolate mononuclear cells, and then centrifuged at $400 \mathrm{~g}$ for 30 minutes at $20^{\circ} \mathrm{C}$. The interface cells were collected and washed three times with the RPMI-1640 medium at $250 \mathrm{~g}$ for 10 minutes. Viability of isolated cells was evaluated by trypan blue exclusion and was determined to be greater than $95 \%$ in each case. The cells were suspended in RPMI-1640 medium containing $10 \%$ foetal calf serum (Sigma-Aldrich) and $1 \%$ antibiotic-antimycotic solution (Sigma-Aldrich), and dispensed into 96-well

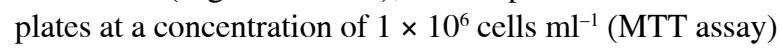
or into 24 -well plates at a concentration of $5 \times 10^{5}$ cells $\mathrm{ml}^{-1}$ (ELISA). Isolated splenocytes consisted of about $90 \%$ of non-adherent cells (lymphocytes) and up to $10 \%$ of adherent cells (phagocytes). Both adherent and non-adherent cells were cultured together in order to maintain the optimal microenvironment for cytokine synthesis and lymphocyte proliferation. The cells were cultured at $37^{\circ} \mathrm{C}$ under a humidified atmosphere of $5 \% \mathrm{CO}_{2}$ and $95 \%$ air atmosphere and used for the following assays.

\section{Proliferative response of splenocytes (MTT assay)}

Mitogenic response of splenocytes was determined using the MTT colorimetric assay [19]. Cells were suspended in RPMI 1640 growth medium containing mitogens: concanavalin A (ConA, Sigma-Aldrich) as a T-cell mitogen or lipopolysaccharide from Escherichia coli (LPS, Sigma-Aldrich) as a B-cell mitogen at concentrations of $5 \mu \mathrm{g} \mathrm{ml}^{-1}$, and $100 \mu \mathrm{l}$ of the suspension was added to each well of microtitre plates. The mixture was incubated for 72 hours in the presence of different concentrations of KYNA (in vitro experiment) or without KYNA (ex vivo experiment). After incubation, $10 \mu \mathrm{l}$ of solution containing $7 \mathrm{mg} \mathrm{ml}^{-1}$ of MTT (3-[4, 5- dimethylthiazoly-2-yl]-2,5-diphenyltetrazolium bromide, Sigma-Aldrich) in PBS were added and 
the plate was incubated for the next four hours. The supernatant was removed and $100 \mu \mathrm{l}$ of dimethyl sulphoxide (DMSO) was added to each well. The optical density (OD) was measured in a Sunrise absorbance reader (Tecan, Austria) at a wavelength of $570 \mathrm{~nm}$ with $640 \mathrm{~nm}$ as a reference wavelength. Samples obtained from each group (in vitro experiment) or each individual (ex vivo experiment) were tested in triplicate. The results of the proliferation assay were expressed as a stimulation index (SI), which was calculated by dividing the mean OD of mitogen stimulated cells by the OD of the non-stimulated cells.

\section{Viability of splenocytes in the presence of KYNA}

MTT assay was also used to determine the viability of splenocytes incubated for 48 hours in the presence of KYNA, without mitogens. In this case the results were expressed as the percentage of the control cell viability.

\section{Determination of cytokine levels}

Murine splenocytes were cultured in the absence (unstimulated cells) or presence (stimulated cells) of LPS from Escherichia coli $\left(5 \mu \mathrm{g} \mathrm{ml}^{-1}\right)$ for 72 hours and centrifuged at $250 \mathrm{~g}$ for 10 minutes, then the supernatants were collected and tested in duplicate. Cytokine levels: interleukin $1 \beta$ (IL-1 $\beta$ ), IL-6, IL-10, and tumor necrosis factor $\alpha$ (TNF- $\alpha$ ) in the culture media were determined using commercial immunoassay (ELISA) kits (R\&D Systems, United Kingdom), according to the manufacturer's protocol. The results were expressed as the relative changes of cytokine concentration in comparison to control cells (in vitro experiment) or the control group of animals (ex vivo experiment). In both cases, control cytokine concentrations (pg/ $\mathrm{ml}$ ) were set to one and the fold increases or decreases in cytokine concentrations for the experimental cells or groups were calculated.

\section{Statistical analysis}

Data are presented as the mean values and the standard deviations. Data were analysed statistically by one-way analysis of variance (ANOVA). Bonferroni's post-test was used to determine differences between groups. Statistical evaluation of results was performed using the GraphPadPrism software package.

\section{Results}

\section{The effect of KYNA on the activity of murine splenocytes under in vitro conditions}

The highest of the tested concentrations of KYNA $(5 \mathrm{mM})$ turned out to be toxic to murine splenocytes under in vitro conditions. This resulted in a significant reduction in both cell viability and cytokine synthesis ability, and impaired their proliferative and cytokine response to mito- gens (Figs. 1A, B, 2A, B). Two of the lower concentrations (2.5 and $1.25 \mathrm{mM}$ ) were also toxic to some extent, reducing the viability of splenocytes (statistically insignificant), inhibiting the proliferation of splenocytes, in particular after ConA-stimulation, and reducing the synthesis of the pleiotropic IL-6 (Fig. 1A, B, 2A, B).

Low, non-toxic concentrations of KYNA $(5 \mu \mathrm{M}-$ $0.5 \mathrm{mM}$ ) had no effect on either the viability of splenocytes (with the exception of the lowest concentration, which exerted a certain proliferative effect) or their proliferative response following the mitogen stimulation (Fig. 1A, B). On the other hand, strong stimulation of cytokine synthesis in the cells non-stimulated by LPS was noted. A wide range of concentrations stimulated the synthesis of IL-1 $\beta(5-250 \mu \mathrm{M})$ and IL-6 (5-125 $\mu \mathrm{M})$, a slightly narrower one $(125 \mu \mathrm{M}$ $0.5 \mathrm{mM}$ ) also stimulated IL-10 and TNF- $\alpha$, whereas the strongest effect was exerted by the concentration of $125 \mu \mathrm{M}$ (Fig. 2A).

Following the stimulation of cells by LPS, the effect of the action of the non-toxic concentrations of KYNA was no longer expressed so widely, and primarily concerned the pleiotropic IL- 6 . The concentrations of 50-250 $\mu \mathrm{M}$ inhibited the synthesis of this cytokine, while the most active concentration of $250 \mu \mathrm{M}$ also reduced the level of proinflammatory IL- $1 \beta$ and TNF- $\alpha$. On the other hand, none of the concentrations modified the synthesis of anti-inflammatory IL-10 (Fig. 2B).

\section{The effect of oral administration of KYNA on the activity of murine splenocytes ex vivo}

None of the doses of KYNA administered to animals in drinking water for a period of one or two weeks had any effect on the proliferative activity of their splenocytes (data not shown). What was noted, however, was a significant impact of kynurenic acid on cytokine synthesis by cells both non-stimulated and stimulated by LPS.

In the non-stimulated cells, all concentrations of KYNA inhibited the synthesis of IL-6, and this effect was more strongly expressed after seven days than after 14 days. After seven days of administration all concentrations also decreased the production of IL-10, and after 14 days this effect was only maintained in the group receiving a medium dose $(25 \mathrm{mg} / \mathrm{l})$. The duration of administration also modified the impact of KYNA on the production of TNF- $\alpha$ by non-stimulated splenocytes. On day seven, the lowest dose $(2.5 \mathrm{mg} / \mathrm{l})$ stimulated, and the medium dose inhibited, the synthesis of this cytokine; on day 14 , the observed effect was only maintained for the medium dose (Fig. 3A, B). Non-stimulated cells did not produce measurable concentrations of IL-1 $\beta$ in any of the animal groups.

After the stimulation by LPS, splenocytes derived from the animals receiving KYNA secreted significantly smaller amounts of IL- $1 \beta$ and IL-6, and larger amounts of anti-inflammatory IL-10, than the cells of animals in the control 
A

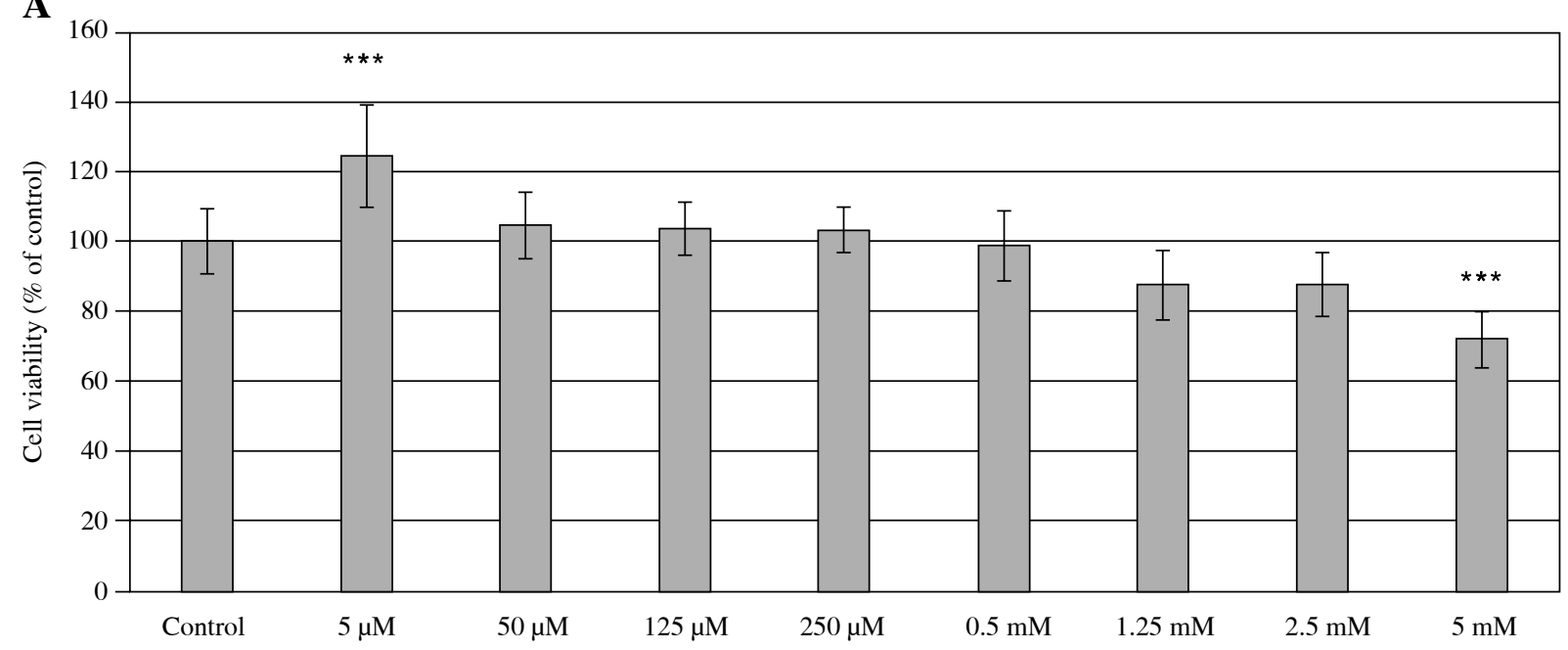

B

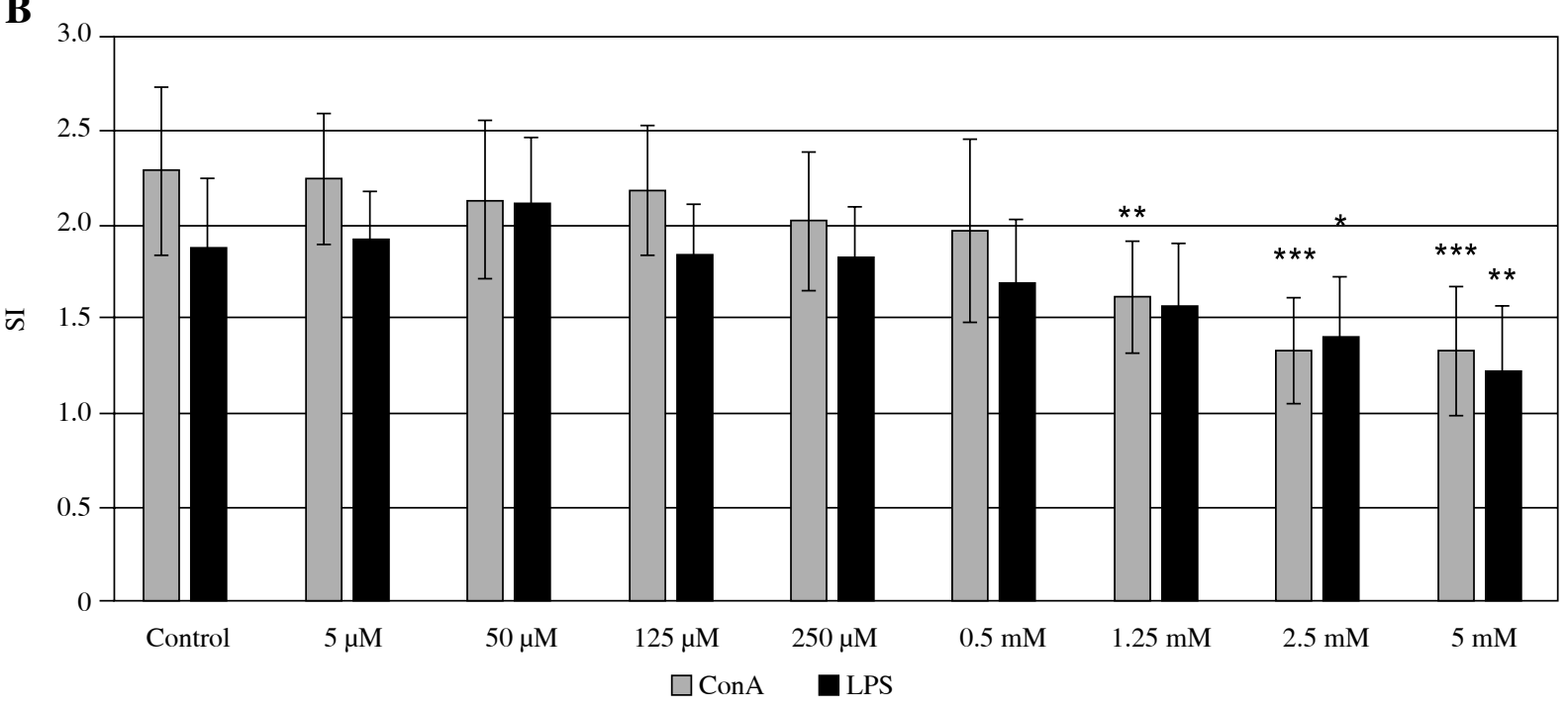

All results are expressed as means $\pm S D$ (standard deviation); the p-values $(* p<0.05, * * p<0.01, * * * p<0.001)$ refer to the significant differences between control cells and experimental cells; $n$ - number of samples; SI-stimulation index; ConA-concanavalin A; LPS - lipopolysaccharide from E. coli

Fig. 1. The in vitro effect of KYNA on the viability (A) and proliferative response (B) of murine splenocytes $(n=8)$

group, irrespective of the duration of administration. On the other hand, the synthesis of TNF- $\alpha$ was modulated with the elongation of the duration of the experiment: after seven days, in the animals receiving KYNA, an elevated level of this cytokine was noted, and after another week, a decrease in its concentration was seen, as compared with the animal control group (Fig. 3C, D).

\section{Discussion}

Under in vitro conditions, KYNA exhibited low toxicity. Only millimolar concentrations exceeding KYNA concentrations noted in a human body in the course of inflammatory processes by hundreds, and even thousands of times, turned out to be toxic to murine splenocytes, lowering their viability and proliferative activity, and decreasing the cytokine response. The obtained results are broadly in line with the results from our previous experiments conducted on immune cells isolated from the peripheral blood and spleen of rainbow trout, as well as on several types of cells unrelated to the immune system (NIH-3T3 mouse fibroblasts, GMK green monkey kidney cells, CECC chick embryo cells). In all the above-mentioned studies, KYNA concentrations up to $1 \mathrm{mM}$ were well tolerated by the cells [20, 21]. Furthermore, a certain proliferative effect noted at the lowest concentration of KYNA remains in compliance with the literature reports and our previously conducted studies. Low, micromolar KYNA concentrations 

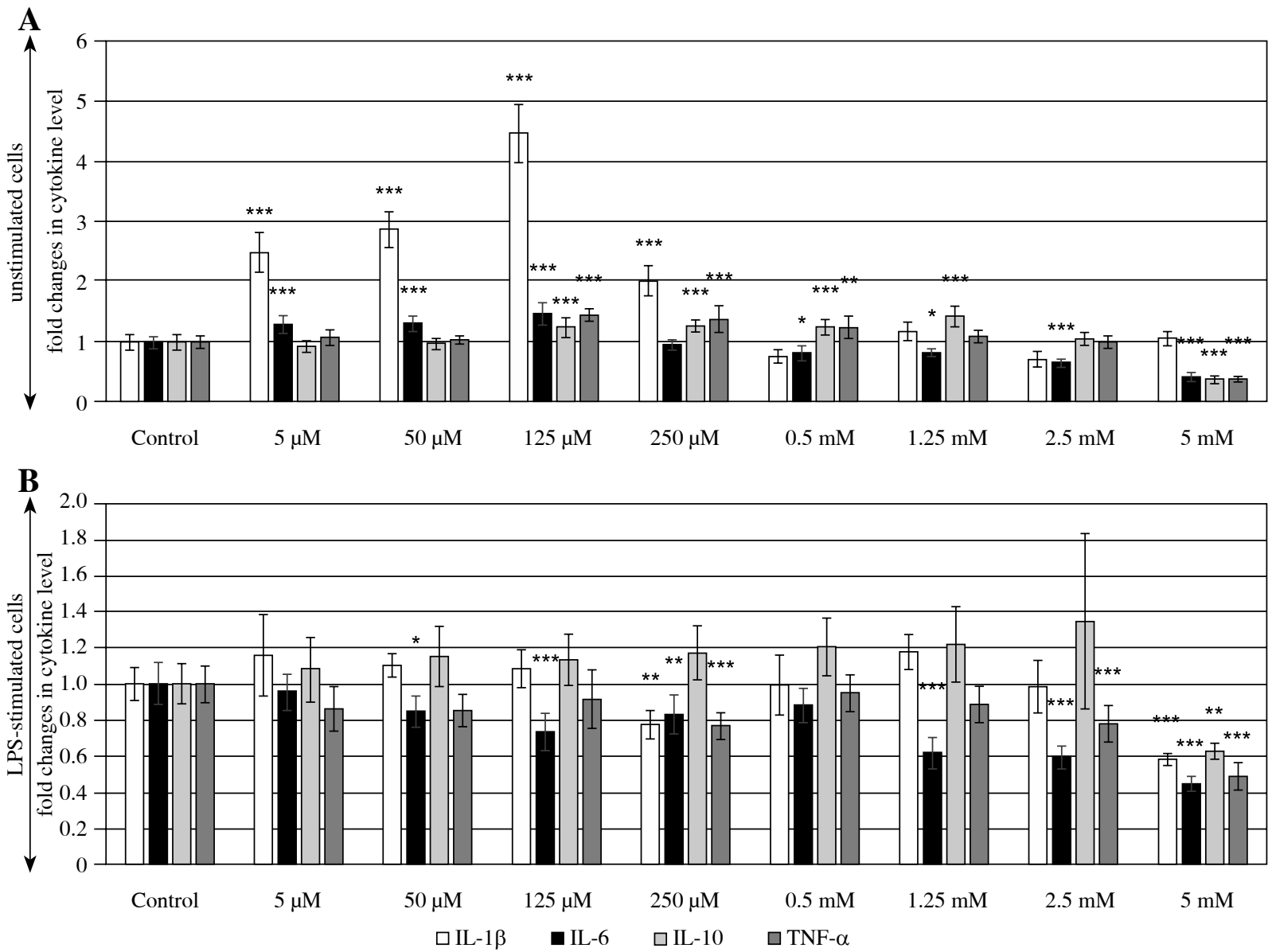

All results are expressed as means $\pm S D$ (standard deviation); the $p$-values $(* p<0.05, * * p<0.01, * * * p<0.001$ ) refer to the significant differences between control cells and experimental cells; $n$ - number of samples; LPS - lipopolysaccharide from E. coli

Fig. 2. The in vitro effect of KYNA on the cytokine production by unstimulated (A) and LPS-stimulated splenocytes (B) $(n=8)$

stimulated the proliferation of fish splenocytes [20], chick embryo fibroblasts, green monkey kidney cells [21], and human and murine glial cells [22, 23].

The mice receiving KYNA in drinking water also tolerated it well. Irrespective of the concentration and the duration of administration, no effects of KYNA on body weight and weight gain rate (data not published), and no changes to haematological parameters, were noted in the animals [21].

The results of all our studies conducted so far indicate immunotropic properties of the exogenous KYNA. It is commonly believed that, similarly to other kynurenines being formed as a result of the stimulation of kynurenine pathway by inflammatory mediators, KYNA is also able, through negative feedback, to exert an antiproliferative effect, particularly towards the stimulated $\mathrm{T}$ lymphocytes [5]. In this study, however, none of the non-toxic concentrations of KYNA under in vitro conditions, and none of the concentrations being received by the animals in drink- ing water had an effect on the proliferative response of murine splenocytes stimulated by mitogens. Similar results under in vitro conditions were obtained by Kudo et al. [3] for PHA-stimulated human peripheral blood mononuclear cells, and by our team for mitogen-stimulated fish immune cells [20]. On the other hand, in our previous studies on animals receiving KYNA at low concentrations with the feed or water, increased proliferation of $\mathrm{T}$ lymphocytes isolated from the peripheral blood of mice, and the spleen of rainbow trout, was noted [10, 24]. These discrepancies may indicate more complex, immunoregulatory properties of kynurenic acid.

Irrespective of the experimental design, KYNA had a significant effect on cytokine synthesis by murine splenocytes; however, we found its different mode of action towards resting, non-stimulated cells under in vitro and ex vivo conditions surprising. In the in vitro experiment, the effect of the action of KYNA changed depending on the functional status of the cells - for resting splenocytes, 
A

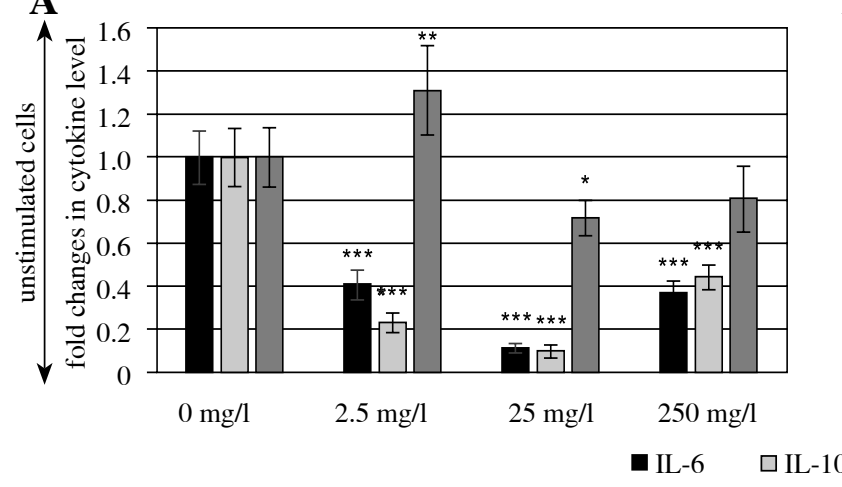

C

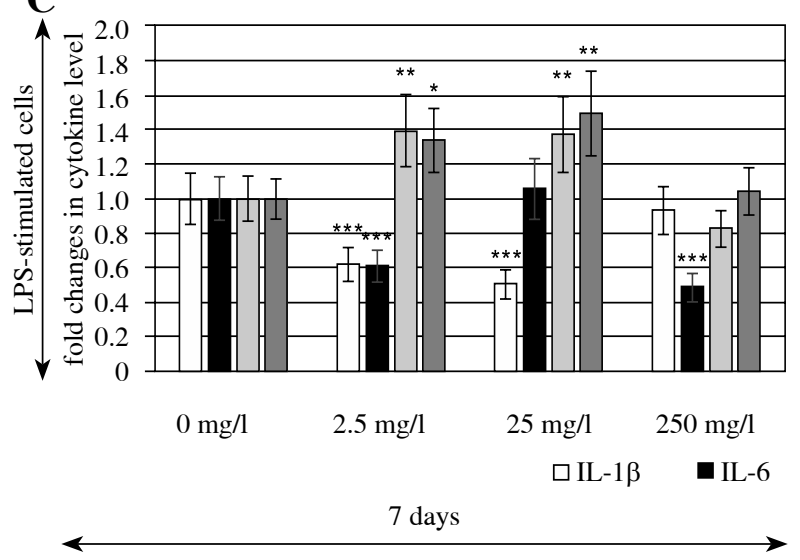

B

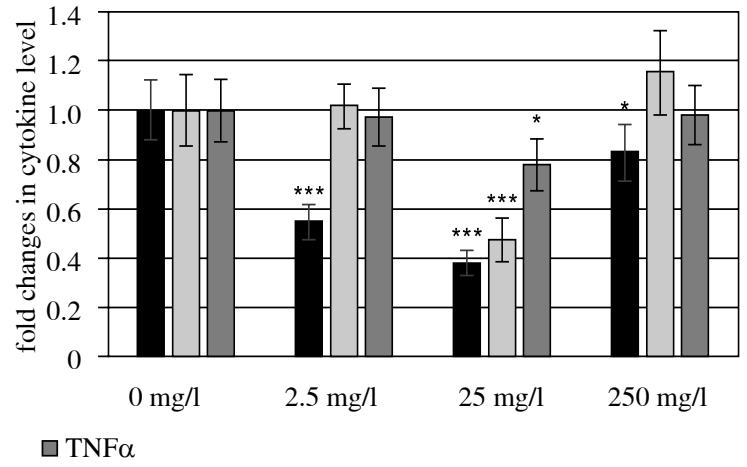

D

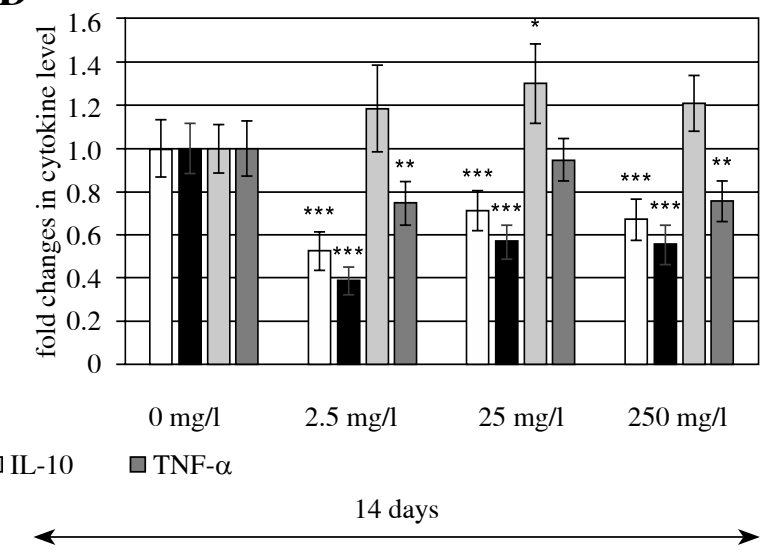

All results are expressed as means $\pm S D$ (standard deviation); the p-values $\left({ }^{*} p<0.05, * * p<0.01, * * * p<0.001\right)$ refer to the significant differences between control group and experimental groups of animals; $n$-number of animals; LPS - lipopolysaccharide from E. coli

Fig. 3. Relative changes in cytokine production by murine splenocytes after oral administration of kynurenic acid $(n=6)$. A, B) Unstimulated cells, administration time 7 and 14 days, respectively; C, D) cells stimulated with bacterial LPS, administration time 7 and 14 days, respectively

a strongly expressed immunostimulation or even proinflammatory effect was noted, while in mitogen-activated cells, KYNA exerted a slightly less pronounced anti-inflammatory effect. Meanwhile, for the animals receiving kynurenic acid with drinking water, its effect underwent certain modifications with the elongation of the duration of administration, but it can generally be described as immunosuppressive for the non-stimulated splenocytes, and anti-inflammatory for the stimulated ones.

The anti-inflammatory action of KYNA towards the activated splenocytes was an expected result, confirming the literature data. Studies by various authors have proven that interactions between KYNA and the GPR35 receptor on the surface of immune cells downregulate the inflammatory response induced by bacterial LPS and other inflammatory stimulators. As a result, a decrease in the level of early (TNF- $\alpha$, IFN- $\gamma$ ) and late (HMGB1) proinflammatory cytokines, and the inhibition of activation of mononuclear peripheral blood cells, granulocytes, macrophages, and glial cells, were noted $[9,11,25,26]$. Bene- ficial effects of KYNA were also demonstrated in vivo on animal models of inflammatory disorders, i.e. acute colitis in rats and enteritis in dogs, and a murine model of septic shock [7, 27, 28]. An important cause of the anti-inflammatory properties of KYNA are its confirmed antioxidant and ROS scavenging properties [1, 7, 8]. A decrease in the levels of IL- $1 \beta$ and TNF- $\alpha$ in stimulated splenocytes under the influence of KYNA in this study could also have resulted from its antioxidative properties because splenic phagocytes are the major ROS producers in this organ as well as stimulators of the inflammatory response, and our previous studies on mice receiving KYNA with drinking water demonstrated a significant decrease in the activity of respiratory burst in peripheral blood granulocytes and monocytes stimulated by $E$. coli, irrespective of the dose and the duration of administration thereof [10].

However, it is the effect of KYNA (in particular ex vivo) on the synthesis of IL-6, probably resulting from its reaction with the AHR receptor, that was expressed most strongly. Even though the effect of KYNA on this receptor 
is less well investigated than on GPR35, the undoubted effect of AHR ligation is a change to the expression of IL-6 in the cell [29]. The final effect of the action of KYNA may vary depending on the type of cells being stimulated. In the mast cells, kynurenic acid, through AHR, decreased the expression of IL- 6 transiently in the non-stimulated cells, and permanently in the stimulated cells [13], similarly to our experiment. Meanwhile, in the MCF-7 breast tumour cells, KYNA induced the expression of IL-6 synergistically with the IL-1 $\beta$ [12].

While this different effect of the action of KYNA on various cell types does not explain the opposite effect of its action on the non-stimulated splenocytes under in vitro and ex vivo conditions as obtained in this study, it provides a basis for discussion on the flexibility of the mode of action of KYNA and its probable dependence on numerous external factors. The results of the in vitro tests performed on isolated cells extracted from the natural cytokine microenvironment and the network of relationships between various types of immune system cells occurring naturally in the body do not necessarily reflect the real mode of action of KYNA in the far more complicated biological system of a living body.

In conclusion, exogenous kynurenic acid was characterised by a low level of cytotoxicity towards murine splenocytes, and was well tolerated by the animals receiving it in drinking water; however, it exhibited immunotropic properties in both experimental designs. As predicted, it exhibited anti-inflammatory action towards the splenocytes activated by LPS, both under in vitro and ex vivo conditions. Surprisingly, however, KYNA itself influenced the activity of resting, unstimulated cells, exerting an immunostimulant effect in vitro, and an immunosuppressive effect under ex vivo conditions. The obtained results indicate more complex, immunomodulating properties of KYNA, which are subject to modification depending on external factors. Drawing any more far-reaching conclusions will require more detailed investigations in this area.

This research was supported by the Polish National Science Centre (grant No NN 308578540).

The authors declare no conflict of interest.

\section{References}

1. Moroni F, Fossati S, Chiarugi A, et al. (2007): Kynurenic acid actions in brain and periphery. Int Congr Ser 1304: 305-313.

2. Chen Y, Guillemin GJ (2009): Kynurenine pathway metabolites in humans: disease and healthy states. Int J Tryptophan Res 2: 1-19.

3. Kudo Y, Boyd CA, Sargent IL, et al. (2001): Tryptophan degradation by human placental indoleamine 2,3-dioxygenase regulates lymphocyte proliferation. J Physiol 535: 207-215.

4. Mándi Y, Vécsei L (2012): The kynurenine system and immunoregulation. J Neural Transm 119: 197-209.

5. Terness P, Bauer TM, Röse L, et al. (2002): Inhibition of allogeneic $\mathrm{T}$ cell proliferation by indoleamine 2,3-dioxygen- ase-expressing dendritic cells: mediation of suppression by tryptophan metabolites. J Exp Med 196: 447-457.

6. Wang J, Simonavicius N, Wu X, et al. (2006): Kynurenic acid as a ligand for orphan $\mathrm{G}$ protein-coupled receptor GPR35. J Biol Chem 281: 22021-22028.

7. Kaszaki J, Palásthy Z, Érczes D, et al. (2008): Kynurenic acid inhibits intestinal hypermotility and xanthine oxidase activity during experimental colon obstruction in dogs. Neurogastroenterol Motil 20: 53-62.

8. Lugo-Huitrón R, Blanco-Ayala T, Ugalde-Muńiz P, et al. (2011): On the antioxidant properties of kynurenic acid: free radical scavenging activity and inhibition of oxidative stress. Neurotoxicol Teratol 33: 538-547.

9. Maes M, Mihaylova I, Ruyter M, et al. (2007): The immune effects of TRYCATs (tryptophan catabolites along the IDO pathway): relevance for depression and other conditions characterized by tryptophan depletion induced by inflammation. Neuro Endocrinol Lett 28: 826-831.

10. Małaczewska J, Siwicki AK, Wójcik RM, et al. (2014): Effect of oral administration of kynurenic acid on the activity of the peripheral blood leukocytes in mice. Cent Eur J Immunol 39: 6-13.

11. Tiszlavicz Z, Németh B, Fülöp F, et al. (2011): Different inhibitory effects of kynurenic acid and a novel kynurenic acid analogue on tumour necrosis factor- $\alpha$ (TNF- $\alpha$ ) production by mononuclear cells, HMGB1 production by monocytes and HNP1-3 secretion by neutrophils. Naunyn Schmiedebergs Arch Pharmacol 383: 447-455.

12. DiNatale B, Murray IA, Schroeder JC, et al. (2010): Kynurenic acid is a potent endogenous aryl hydrocarbon receptor ligand that synergistically induces interleukin- 6 in the presence of inflammatory signaling. Toxicol Sci 115: 89-97.

13. Maaetoft-Udsen K, Shimoda LM, Frřkićr H, et al. (2012): Aryl hydrocarbon receptor ligand effects in RBL2H3 cells. J Immunotoxicol 9: 327-337.

14. Paluszkiewicz P, Zgrajka W, Saran T, et al. (2009): High concentration of kynurenic acid in bile and pancreatic juice. Amino Acids 37: 637-641.

15. Zgrajka W, Turska M, Rajtar G, et al. (2013): Kynurenic acid content in anti-rheumatic herbs. Ann Agric Environ Med 20: 800-802.

16. Turski MP, Turska M, Zgrajka W, et al. (2009): Presence of kynurenic acid in food and honeybee products. Amino Acids 36: $75-80$

17. Turski MP, Turska M, Paluszkiewicz P, et al. (2013): Kynurenic acid in the digestive system - new facts, new challenges. Int J Tryptophan Res 6: 47-55.

18. Wejksza K, Rzeski W, Turski WA (2009): Kynurenic acid protects against the homocysteine-induced impairment of endothelial cells. Pharmacol Rep 61: 751-756.

19. Mosmann T (1983): Rapid colorimetric assay for cellular growth and survival: application to proliferation and cytotoxicity assays. J Immunol Methods 65: 55-63.

20. Małaczewska J, Siwicki AK, Wójcik R, et al. (2014): The in vitro effect of kynurenic acid on the rainbow trout (Oncorhynchus mykiss) leukocyte and splenocyte activity. Pol J Vet Sci 17: 453-458.

21. Turski WA, Małaczewska J, Marciniak S, et al. (2014): On the toxicity of kynurenic acid in vivo and in vitro. Pharm Rep 66: 1127-1133.

22. Klien C, Patte-Mensah C, Taleb O, et al. (2013): The neuroprotector kynurenic acid increases neuronal cell survival 
through neprilysin induction. Neuropharmacology 70: 254260.

23. Di Serio C, Cozzi A, Angeli I, et al. (2005): Kynurenic acid inhibits the release of the neurotrophic fibroblast growth factor (FGF)-1 and enhances proliferation of glia cells, in vitro. Cell Mol Neurobiol 25: 981-993.

24. Małaczewska J, Siwicki AK, Wójcik R, et al. (2013): Effect of dietary administration of kynurenic acid on the activity of splenocytes of the rainbow trout (Oncorhynchus mykiss). Centr Eur J Immunol 38: 475-479.

25. Kiank C, Zeden JP, Drude S, et al. (2010): Psychologial stress-induced, IDO1-dependent tryptophan catabolism: implications on immunosuppression in mice and humans. PLoS One 5: e11825.

26. Steiner L, Gold M, Mengel D, et al. (2014): The endogenous $\alpha 7$ nicotinic acetylcholine receptor antagonist kynurenic acid modulates amyloid- $\beta$-induced inflammation in BV-2 microglial cells. J Neurol Sci 344: 94-99.

27. Moroni F, Cozzi A, Sili M, et al. (2012): Kynurenic acid: a metabolite with multiple actions and multiple targets in brain and periphery. J Neural Transm 119: 133-139.

28. Varga G, Erces D, Fazekas B, et al. (2010): N-methyl-D-aspartate receptor antagonism decreases motility and inflammatory activation in the early phase of acute experimental colitis in the rat. Neurogastroenterol Motil 22: 217-225.

29. Stone TW, Stoy N, Darlington LG (2013): An expanding range of targets for kynurenine metabolites of tryptophan. Trends Pharmacol Sci 34: 136-143. 